\title{
Potential adverse effects of amphetamines on kidney; a narrative review on current knowledge
}

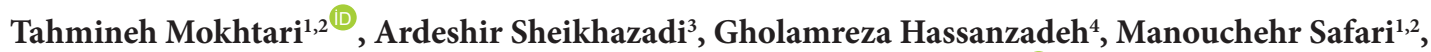 \\ Fatemeh Sheikhbahaei ${ }^{5}$, Hedyeh Faghir-Ghanesefat ${ }^{6}$, Maryam Rezaei $^{{ }^{*} \mathbb{C}}$

\begin{abstract}
${ }^{1}$ Nervous System Stem Cells Research Center, Semnan University of Medical Sciences, Semnan, Iran ${ }^{2}$ Department of Anatomy, School of Medicine, Semnan University of Medical Sciences, Semnan, Iran ${ }^{3}$ Department of Forensic Medicine and Toxicology, School of Medicine, Tehran University of Medical Sciences, Tehran, Iran ${ }^{4}$ Department of Anatomy, School of Medicine, Tehran University of Medical Sciences, Tehran, Iran

${ }^{5}$ Department of Anatomical Sciences, Afzalipour School of Medicine, Kerman University of Medical Sciences, Kerman, Iran

${ }^{6}$ Department of Pharmacology, School of Medicine, Tehran University of Medical Sciences, Tehran, Iran
\end{abstract}

\section{A R T I C L E I N F O}

Article Type:

Review

\section{Article History:}

Received: 9 October 2017

Accepted: 8 December 2017

Published online: 20 January 2018

\section{Keywords:}

Acute kidney injury,

Amphetamines, Kidney, Drug

abuse, Nephropathy, Necrotizing

renal vasculopathy

\begin{abstract}
A B S T R A C T
This study aims to review the literature pertaining to the renal effects of amphetamines (AMPs) including AMP and its analogues (ARDAs) such as methamphetamine (MET) and 3,4-methylenedioxy- $N$-methylamphetamine (MDMA) and discuss the implications for these drugs users. The study was performed through searching in the relevant literature. Sufficient clinical and experimental evidence suggested that AMPs have adverse and potentially nonfatal and fatal effects on users' kidneys. The acute and chronic renal complications due to illicit drugs such as AMPs have been investigated. A few renal effects in AMPs users have been demonstrated. However, isolated reports of adverse renal effects of AMPs including nephropathy due to rhabdomyolysis, necrotizing renal vasculopathy and malignant hypertension have been recorded. It can be suggested that renal damages after AMPs consumption should be considered in the emergency departments (EDs).
\end{abstract}

Implication for health policy/practice/research/medical education:

Isolated reports on renal complications, rhabdomyolysis, necrotizing renal maculopathy and malign hypertension, induced by amphetamine (AMP) and its analogues have been recorded. This review tried to survey different sources to collect information for better understanding the pathophysiology underlying the functional defects and different renal complications in patients with AMP abuse.

Please cite this paper as: Mokhtari T, Sheikhazadi A, Hassanzadeh G, Safari M, Sheikhbahaei F, Faghir-Ghanesefat H, et al. Potential adverse effects of amphetamines on kidney; a narrative review on current knowledge. J Renal Inj Prev. 2018;7(4):218223. Doi: 10.15171/jrip.2018.51.

\section{Introduction}

Drug abuse is a major health concern of countries nowadays. According to the clinical observations, the prevalence of addiction to new psychoactive drugs is increasing among the adolescence and youth in Iran (1). In the United States, the lifetime use of illicit drug was reported $47.0 \%$ of respondents (with more than 12 years of age) according to the 2011 National Survey on Drug Use and Health (NSDUH) (2). Moreover, studies have found that the age range of drug abusers has decreased all over the world which raised deep concern in different communities (3).

Among the illicit drugs, amphetamines (AMPs) with significant adverse physical effects remain popular recreationaldrugintheWorld.OverdoseandillicitAMPsand its analogues (ARDAs) such as methamphetamine (MET), ecstasy or 3,4-methylenedioxy- $N$-methylamphetamine (MDMA), ephedrine, pseudoephedrine, methylphenidate and lisdexamfetamine is an international growing problem. Also, 50 million ongoing AMP users were estimated based on United Nations World Drug Report (4).

According to the reports from the Substance Abuse and 
Mental Health Services Administration (SAMHSA), more than 150000 emergency department (ED) visits were described for toxicity from ARDA in 2010 for acute care (5). Furthermore, a wide range of diagnoses such as sepsis, acute psychosis, pheochromocytoma, thyrotoxicosis, anticholinergic toxicity, alcohol, benzodiazepine and opioid withdrawals, intracranial hemorrhage and neuroleptic malignant syndromes must be considered during the initial face-to-face evaluation (6).

Amphipathic molecules can cross the placenta and blood-brain barrier and enhance concentrations of norepinephrine, serotonin and dopamine through multiple mechanisms (7). Elevated levels of monoamines cause blockade of plasmalemmal and vesicular transporters in the cytoplasm and synapse, respectively. Hence, the reverse transport of cytoplasmic monoamines across the cell membrane of the presynaptic neuron into the synaptic space happens. AMP and its analogues also inhibit the degradative enzymes (monoamine oxidase A and B) and disrupt vesicular storage of monoamines. Suddenly, the serum catecholamines rise and heart rate (HR), systolic blood pressure (SBP), and diastolic blood pressure (DBP) unpredictably will increase (8). This potential hyperadrenergic effect of AMPs has been observed with varying degrees based on the specific ARDA, patient's tolerance, pharmacogenetics and source of administration (9).

In small doses (5-10 mg), AMPs can improve concentration and capacity for physical effort, while excessive dosage of AMPs reduce appetite, raise body temperature and arterial blood pressure, cause insomnia, increased excitability, euphoria, trembling, headaches and dizziness, dryness in the mouth, perspiration, tachycardia and difficulties with micturition agitation, anxiety, hallucinations, psychosis, seizures and coma $(10,11)$. People under the influence of AMPs present an increased motor and mental activity, without feeling sleepy or tired (11). Also, cardiovascular symptoms associated with the toxic effects of AMPs have been recorded $(12,13)$.

The acute and chronic renal complications due to illicit drugs have been mentioned. It has been estimated that substance-related renal diseases may involve 5\%-6\% of new patients starting end-stage renal disease (ESRD) therapy in the United States (14). No renal effects in AMPs users have been mentioned in a review of the histopathological findings (15). However, isolated reports of adverse renal effects of AMPs including necrotizing renal vasculopathy have been recorded $(16,17)$.

Since that it is difficult to perform controlled prospective studies in substance abusers, the adverse effects of AMPs on kidney are difficult to assess. The current article reviews the current knowledge about AMPs -related renal pathology and discusses implications for AMPs abusers.

Materials and Methods

For this review, we used a variety of sources by searching

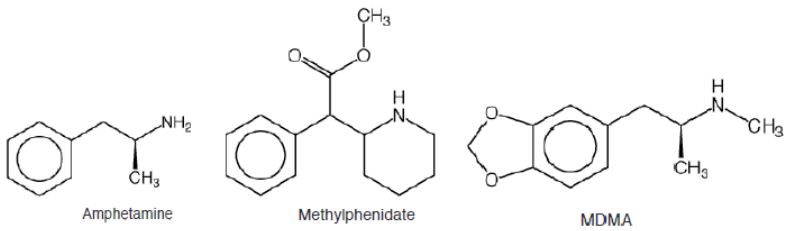

Figure 1. Chemical structure of amphetamine, an MDMA (18).

through AMP and the most common ARDA (MDMA and MET) were selected to discuss (their chemical structure was shown in Figure 1). In this way, searches of different sources such as Web of Science, PubMed, Embase, Scopus and directory of open access journals (DOAJ) and other relevant materials were conducted. The search was performed using combinations of the following key words and or their equivalents such as AMP, MDMA, MET, drug abuse and keywords related to renal pathology (such as renal failure, necrotizing renal vasculopathy, rhabdomyolysis, malignant hypertension, methamphetamine, nephropathy, acute renal failure, catecholamines, hyperadrenergic state, Acute kidney injury, End-stage renal failure, kidney and nephrotoxicity).

\section{Renal pathology associated with ARDA abuse Adverse effects of AMP on kidney}

AMP synthetized from phenethylamine stimulants central nervous system and release catecholamines (like norepinephrine and dopamine) from presynaptic nerve terminals and also inhibit their reuptake, thus potentiates their synaptic effects (19). After AMP using, the circulatory system rapidly absorbs and transports it. It can be present in the urine within 20 minutes and about $20 \%-30 \%$ of the dose with no changes is excreted with the urine. After excessive dosage, AMP can be observed in the urine for a few days (11). The renal complications as a result of AMP use are associated with hyperpyrexia and fibrinolysis (disseminated intravascular coagulation; DIC). Also, microvascular obstruction secondary to DIC, myoglobinuria, systemic hypotension or hyperpyrexia may lead to acute renal failure (ARF) (20).

Different isolated case reports were mentioned the adverse effects of AMP on kidney. AMP induces coexisting hyperpyrexia or rhabdomyolysis in AMP abusers. Rhabdomyolysis with a wide range of clinical manifestations and life-threatening complications is characterized by breakdown of the muscles and release of the intracellular contents (CPK, LDH and transaminases, among other components) of the muscle into the systemic circulation $(21,22)$. Additionally, a relationship between rhabdomyolysis due to AMF and ARF, known as the pigment nephropathy. First ARF secondary to AMP was described by Ginsberg et al in 1970. In this case report, a 21-year-old who took approximately $2 \mathrm{~g}$ of AMP sulfate was argued. A range of complications such as temperature of above $108 \mathrm{~F}$, coagulopathy with intramuscular 
hemorrhages, developed acute self-limited renal failure and an entrapment neuropathies were found in this patient (23). Heras et al described a case of a young man with severe complications such as ARF associated with an extreme rhabdomyolysis due to a physical aggression aggravated by the consumption of AMP. The electrolyte disorders with electrocardiographic repercussion and the ARF were observed in this case of study (24). Based on the study by Chen et al, among the clinical factors that predicted the occurrence of ARF, an initial value of $600 \mathrm{ng} /$ $\mathrm{dL}$ of myoglobin was a predictor of ARF (25). Wiergowski et al described a 31-year old man with acute poisoning resulted from the use of methoxetamine (MXE) and AMP and ended in death. Massive rhabdomyolysis, hepatic failure and ARF were reported due to laboratory tests. They noted a possibility of adverse interactions between AMP and MXE in this patient (11). However, Foley et al introduced a 32-year-old man with ARF after ingesting AMPs. Based on pathological findings, acute interstitial nephritis was reported in this case. In contrast to other reports, the results supported that acute renal injury resulted to AMP alone (26).

\section{Adverse effects of MET on kidney}

In 1893, MET was first synthesized in Japan as a methylated analogue of AMP. The substance abuse of MET was common during World War II to improve the energy of American, German and Japanese soldiers (4). In the 1990s, MET was established popular in the United States and introduced into European countries (27). Its mechanism of action is similar to AMP and stimulates the secretion of catecholamines (serotonin and norepinephrine and mostly dopamine) from nerve endings and prevent reuptake of neurotransmitters and create a hyperadrenergic state (28). A wide spectrum of histopathological changes as well as laboratory findings such as enhanced creatinine levels in biopsies after death were suggested in the cases of MET poisoning (29-31). According to the United Nations Office on Drugs and Crime (UNODC) estimations, Iran ranked fifth in MET seizure in the worldwide (32).

There was no report on direct effects of MET and its active metabolites on renal tubules. Additionally, ARF due to MET consumption is often associated with the hyperthermia and/or hemodynamic instability (28). Early hemodynamic instability caused by volume depletion can result in prerenal ARF and its severe and prolonged impacts can result in ischemic acute tubular necrosis (33). MET also lead to rhabdomyolysis and related nephropathies. Acute MET intoxication in 18 patients (male: female ratio, 11:7) during a 6-year period was investigated in the study by Lan et al. Higher levels of blood urea nitrogen and serum creatinine and lower values of arterial $\mathrm{pH}$ were observed in that expired patients compared to cases who survived. The most common complication in the dead cases was rhabdomyolysis with ARF (5 of 5) from acute MET intoxication (34). Richards et al stated that $43 \%$ of patients (166 cases) identified with rhabdomyolysis were positive teste for MET in a five year retrospective study of patients admitted to an ED. They recommended that patients diagnosed with rhabdomyolysis with an unclear cause should be screened for MET or other illicit substances. Although the initial mean levels of creatine phosphokinase (CPK) were higher in patients with MET toxicity, mean peak levels were lower than patients with no drug abuse (35). In 2003, a research was investigated to detect the myoglobin and oxidative injury-related factors in the kidney of 22 cases with MET consumption. Their research found that 17 cases were positive for myoglobin and the $\mathrm{Cu} / \mathrm{Zn}$ superoxide dismutase (SOD), 8-hydroxy-2'-deoxyguanosine (8-OH-dG), $70 \mathrm{kDa}$ heat shock protein (HSP70), 4-hydroxy-2-nonenal (4-HNE) were also positive in some cases (36). To confirm the results of this investigation, an animal study conducted by Tokunaga et al and renal function and oxidative damage from MET was evaluated in rats. By repeated administration of MET, oxidative DNA and cell damages were observed in immunohistochemistry studies. Elevated creatinine, myoglobin and $\mathrm{CPK}$ and decreased $\mathrm{K}, \mathrm{Ca}$, and $P$ levels in blood were also recorded. In this study, renal dysfunction due to renal tubular damage was linked to MET consumption (37).

A systemic disease similar to a medium vessel vasculitis was described in 1970 for patients who most commonly used intravenous MET among a 14 chronic users of stimulants, opiates, hallucinogens and depressants. In these patients, a variety of diseases such as acute kidney injury, hypertension and neuropathy were reported. Moreover, their angiography discovered arterial aneurysms in different organs such as small intestine, liver, pancreas and kidneys (38). In addition, Jones et al reviewed 47 patients referred to a renal unit with a history of MET use. Chronic kidney disease (CKD) was observed in $95.7 \%$ of patients and also $55.3 \%$ had ESRD. Renal biopsies, performed in 24 patients, proved different alterations such as mesangiocapillary glomerulonephritis, type 1 (with deposition of C3 complement and IgM) in 14 patients and hypertensive changes in 12 patients (39). Vupputuri et al evaluated the kidney function of 647 patients regarding illicit drug use and followed up for a median of seven years to estimate the incidence of decline in kidney function (increase in serum creatinine level $>0.6$ $\mathrm{mg} / \mathrm{dL}$ ). Different drugs were recorded for participants including marijuana (22.7 \%), AMPs (9.3\%), cocaine or crack $(6.7 \%)$, heroin $(4.3 \%)$ and psychedelics $(3.1 \%)$. Their findings study indicated that there was a significant association between illicit substance abuse and risk of decline in renal function (17). Also, in a study by Inouye et al, an elevated serum creatinine concentration was observed one year after kidney transplant in recipients from donors with MET users (40).

\section{Adverse effects of MDMA on kidney}

A 'party' drug, MDMA, known as ecstasy, was first 
synthesized in 1914. In 2010, 10-28 million people worldwide used the MET derivative MDMA, based on UNODC estimations (4). It is usually used at nightclubs by groups of young people rather than by single users (41). In a similar mechanism to AMP, MDMA stimulates the secretion of neurotransmitters (serotonin, dopamine and noradrenaline. Also, by inhibiting the neurotransmitters reuptake, it increases their concentration at the synapses and leads to acute neurological defects (42).

One oral dosage of MDMA is rapidly absorbed through the gastrointestinal tract and the time to reach the peak concentration is 2 hours (42). It takes approximately 40 hours to be cleared from the body because of slow elimination of MDMA and its active metabolites (43). Symptomatic hyponatremia, the most common renal complication of MDMA use, is associated with hyponatremia deaths. Hyponatremia is due to water balance impairment after MDMA ingestion which induced by secretion of arginine vasopressin (AVP). Moreover, polydipsia with high intake of fluids leads to hyponatremia as a result of MDMA effects on the serotonergic nervous pathways (42). After MDMA consumption, the systemic effects of MDMA can induce nephrotoxicity pigment nephropathy from rhabdomyolysis is the most common cause of ARF in users of MDMA, mentioned in several studies (44). MDMA has been linked with necrotizing vasculitis in the small arteries. In one patient (30 years old) described by Bingham et al, acute kidney injury leading to ESRD was proven after use of oral MDMA and MET. He remained on hemodialysis and the analysis of the renal biopsies confirmed the involvement of arterioles. From with fibrinoid necrosis of arterioles and glomeruli with focal segmental to edematous intimal thickening with severe vasculitis necrosis was seen in the biopsies (16). Hurault et al investigated early graft loss of two kidneys secondary to necrotizing vasculitis. The donor (a 21-yearold woman) had regularly used MDMA over a two-year period. In this study, MDMA was suggested as possible cause of necrotizing vasculitis in the donor kidneys leading to these two renal-graft losses (10). Hyperpyrexia and fluid loss resulting in ingestion of MDMA may be associated with renal damage. Although it was not associated with obstructive nephropathy or ARF, Bryden et al stated that MDMA misuse also developed urinary retention in the short term (45).

Renal proximal tubular injury has been associated with the MDMA use. Another interesting case study by Kwon et al introduced an 18-year-old woman with oral use of MDMA. Her laboratory evaluation presented hyponatremia and polyuria, renal glycosuria, phosphaturia and solute diuresis. Their findings asserted an acute and transient proximal tubular injury associated with possible direct effects of MDMA on kidney (46). Increased oxidative stress or mitochondrial dysfunction due to MDMA may induce tubular damages in kidney tissue (47). MDMA metabolism and/or toxic oxidation elevate the reactive oxygen species (ROS) in the organ and are responsible for tissue damage to the kidneys (48). In support of this finding, Ninković et al demonstrated that MDMA induced oxidative stress in rat kidneys. Their findings revealed the increased SOD activity and thiobarbituric acid reactive substances in renal tissue in subacute MDMA-administered rats. Histopathological studies showed a dose-dependent $(5,10$ or $20 \mathrm{mg} / \mathrm{kg}$ over 14 days) disruption of renal structure after MDMA administration (49).

\section{Conclusion}

Accompanying health impacts of AMPs will become more common in the future due to an increase in the prevalence of young people with elicit use of these drugs. Induced adverse effects of AMPs including mortality, particularly in young patients with serious, hypertension, hyperthermia or hyponatremia are discussable. The deadly side effects of AMPs should be considered in the clinic. One of the more important complications of the AMPs is associated with their renal defects due to rhabdomyolysis, necrotizing renal maculopathy and malign hypertension. Therefore, nephropathy after AMPs consumption should be considered in the EDs.

\section{Authors' contribution}

TM and MR conducted the searches. TM, AS, MR and FS searched and gathered the related articles. TM, MS and NB prepared the draft. MR and HG edited the final manuscript. All authors read and signed the final paper.

\section{Conflicts of interest}

The authors declare no conflicts of interest.

\section{Ethical considerations}

Ethical issues (including plagiarism, data fabrication, double publication) have been completely observed by the authors.

\section{Funding/Support}

None

\section{References}

1. Geramian N, Gharaat L, Taheri SA, Mohebpour F, Nahvizadeh M, Farajzadegan Z, et al. Development of a questionnaire to assess drug abuse among high school students of Isfahan province, Iran: An action research. Int J Prev Med. 2014;5:S146. doi: 10.4103/2008-7802.157678.

2. Crane EH. Mental Health Services Administration, Drug Abuse Warning Network, 2011: National Estimates of Drug-Related Emergency Department Visits. The CBHSQ Report. Rockville (MD): Substance Abuse and Mental Health Services Administration (US); 2013.

3. Rezaei A, Delavar A, Najafi M. The Construction and validation of opium attitude questionnaire among guidance and high school students. J Res Addict. 2012;6:37-54.

4. United Nations Office on Drugs and Crime. World Drug Report 2013. http://www.unodc.org/unodc/secured/wdr/ 
wdr2013/World_Drug_Report_2013.pdf.

5. Substance Abuse and Mental Health Services Administration: Results from the 2008 National Survey on Drug Use and Health: National Findings. Rockville, Md, SAMHSA, 2010. http://www.dpft.org/resources/ NSDUHresults2008.pdf.

6. Richards JR, Albertson TE, Derlet RW, Lange RA, Olson KR, Horowitz BZ. Treatment of toxicity from amphetamines, related derivatives, and analogues: A systematic clinical review. Drug Alcohol Depend. 2015;150:1-13. doi: 10.1016/j.drugalcdep.2015.01.040.

7. Panenka WJ, Procyshyn RM, Lecomte T, MacEwan GW, Flynn SW, Honer WG, et al. Methamphetamine use: a comprehensive review of molecular, preclinical and clinical findings. Drug Alcohol Depend. 2013;129:167-79. doi: 10.1016/j.drugalcdep.2012.11.016.

8. Fleckenstein AE, Volz TJ, Riddle EL, Gibb JW, Hanson GR. New insights into the mechanism of action of amphetamines. Annu Rev Pharmacol Toxicol. 2007;47:68198. doi: 10.1146/annurev.pharmtox.47.120505.105140.

9. De La Torre R, Yubero-Lahoz S, Pardo-Lozano R, Farré M. MDMA, methamphetamine, and CYP2D6 pharmacogenetics: what is clinically relevant? Front Genet. 2012;3:235. doi: 10.3389/fgene.2012.00235.

10. de Ligny BH, El Haggan W, Comoz F, Lobbedez T, Pujo M, Griveau AM, et al. Early loss of two renal grafts obtained from the same donor: role of ecstasy? Transplantation. 2005;80:153-6.

11. Wiergowski M, Anand J, Krzyżanowski M, Jankowski Z. Acute methoxetamine and amphetamine poisoning with fatal outcome: a case report. Int J Occup Med Environ Health. 2014;27:683-90. doi: 10.2478/s13382-014-0290-8.

12. Ito H, Yeo KK, Wijetunga M, Seto TB, Tay K, Schatz IJ. A comparison of echocardiographic findings in young adults with cardiomyopathy: with and without a history of methamphetamine abuse. Clin Cardiol. 2009;32:E18-22. doi: 10.1002/clc.20367.

13. Ito H, Yeo KK, Wijetunga M, Seto TB, Tay K, Schatz IJ. A comparison of echocardiographic findings in young adults with cardiomyopathy: with and without a history of methamphetamine abuse. Clin Cardiol. 2009;32:E18-22. doi: $10.1002 /$ clc.20367.

14. Orth SR. Adverse renal effects of legal and illicit drugs. Ther Umsch. 2002;59:122-30. doi: 10.1024/0040-5930.59.3.122.

15. Milroy CM, Parai JL. The histopathology of drugs of abuse. Histopathology. 2011;59:579-93. doi: 10.1111/j.13652559.2010.03728.x.

16. Bingham C, Beaman M, Nicholls AJ, Anthony PP. Necrotizing renal vasculopathy resulting in chronic renal failure after ingestion of methamphetamine and 3, 4-methylenedioxymethamphetamine ('ecstasy'). Nephrol Dial Transplant. 1998;13:2654-5.

17. Vupputuri S, Batuman V, Muntner P, Bazzano LA, Lefante JJ, Whelton PK, et al. The risk for mild kidney function decline associated with illicit drug use among hypertensive men. Am J Kidney Dis. 2004;43:629-35. doi: 10.1053/j. ajkd.2003.12.027.

18. Richards J, Derlet R, Albertson T, Horowitz B, Lange R. Methamphetamine,"bath salts," and other amphetaminerelated derivatives: progressive treatment update. Enliven Toxicol Allied Clin Pharmacol. 2014;1:001.

19. Yamamoto BK, Moszczynska A, Gudelsky GA. Amphetamine toxicities: classical and emerging mechanisms. Ann N Y Acad Sci. 2010;1187:101-21. doi: 10.1111/j.1749-6632.2009.05141.x.

20. Stewart $H$. Acute renal failure after amphetamine presenting with loin pain. Br J Urol. 1998; 81:160-1.

21. Cervellin G, Comelli I, Lippi G. Rhabdomyolysis: historical background, clinical, diagnostic and therapeutic features. Clin Chem Lab Med. 2010;48:749-56. doi: 10.1515/ CCLM.2010.151.

22. Zutt R, van der Kooi AJ, Linthorst GE, Wanders RJ, de Visser M. Rhabdomyolysis: a review of the literature. Neuromuscul Disord. 2014;24:651-9. doi: 10.1016/j. nmd.2014.05.005.

23. Ginsberg MD, Hertzman M, Schmidt-Nowara WW. Amphetamine intoxication with coagulopathy, hyperthermia, and reversible renal failure: a syndrome resembling heatstroke. Ann Intern Med. 1970;73:81-5.

24. Heras M, Callejas R, Rodríguez MA, Molina Á, FernándezReyes MJ. Rhabdomyolysis after physical aggression and aggravated by amphetamines. Rev Colomb Reumatol. 2016;23:44-6. doi: 10.1016/j.rcreue.2016.04.004

25. Chen C-Y, Lin Y-R, Zhao L-L, Yang W-C, Chang Y-J, Wu H-P. Clinical factors in predicting acute renal failure caused by rhabdomyolysis in the ED. Am J Emerg Med. 2013;31:1062-6. doi: 10.1016/j.ajem.2013.03.047.

26. Foley RJ, Kapatkin K, Verani R, Weinman E. Amphetamineinduced acute renal failure. South Med J. 1984;77:258-60.

27. C Mariotti K, G Rossato L, E Froehlich P, P Limberger R. Amphetamine-type medicines: a review of pharmacokinetics, pharmacodynamics, and toxicological aspects. Curr Clin Pharmacol. 2013;8:350-7.

28. Schep LJ, Slaughter RJ, Beasley DMG. The clinical toxicology of metamfetamine. Clin Toxicol(Phila). 2006;44:379-82.

29. Maeda H. [Pathophysiochemistry of acute death: an approach to evidence-based assessment in forensic pathology]. Nihon Hoigaku Zasshi. 2004;58:121-9.

30. Richards JR. Rhabdomyolysis and drugs of abuse. J Emerg Med. 2000;19:51-6. doi: 10.1016/S0736-4679(00)00180-3.

31. Kuroda R, Nakajima M, Nagai H, Maeda H, Kashima J, Saka $\mathrm{K}$, et al. Small focal coagulation necrosis in the myocardium of a young methamphetamine user. Int J Cardiol. 2013; 20;164:e5-6. doi: 10.1016/j.ijcard.2012.09.145.

32. Amini N, Etemadi-Aleagha A, Akhgari M. Impurity profiling of street methamphetamine samples seized in Kermanshah, Iran with special focus on methamphetamine impurities health hazards. J Clin Toxicol. 2015;5:1-7. doi: 10.4172/2161-0495.1000258

33. Ago M, Ago K, Hara K, Kashimura S, Ogata M. Toxicological and histopathological analysis of a patient who died nine days after a single intravenous dose of methamphetamine: a case report. Leg Med (Tokyo). 2006;8:235-9. doi: 10.1016/j. legalmed.2006.03.002.

34. Lan K-C, Lin Y-F, Yu F-C, Lin C-S, Chu P. Clinical manifestations and prognostic features of acute methamphetamine intoxication. J Formos Med Assoc. 1998;97:528-33.

35. Richards JR, Johnson EB, Stark RW, Derlet RW. Methamphetamine abuse and rhabdomyolysis in the ED: a 5-year study. Am J Emerg Med. 1999;17:681-5.

36. Ishigami A, Tokunaga I, Gotohda T, Kubo S-i. Immunohistochemical study of myoglobin and oxidative injury-related markers in the kidney of methamphetamine abusers. Leg Med (Tokyo). 2003;5:42-8. doi: 10.1016/ S1344-6223(03)00005-1. 
37. Tokunaga I, Kubo S-i, Ishigami A, Gotohda T, Kitamura O. Changes in renal function and oxidative damage in methamphetamine-treated rat. Legal Med. 2006;8:16-21. doi: 10.1016/j.legalmed.2005.07.003.

38. Citron BP, Halpern M, McCarron M, Lundberg GD, McCormick R, Pincus IJ, et al. Necrotizing angiitis associated with drug abuse. N Engl J Med. 1970;283:100311. doi: 10.1056/NEJM197011052831901.

39. Jones ESW, Rayner BL. Hypertension, end-stage renal disease and mesangiocapillary glomerulonephritis in methamphetamine users. S Afr Med J. 2015;105:199-201.

40. Inouye DS, Kickertz K, Wong LL. Methamphetamine use in deceased kidney donors impairs one-yr graft function. Clin Transplant. 2007;21:643-50. doi: 10.1111/j.13990012.2007.00703.x.

41. Kalant $H$. The pharmacology and toxicology of "ecstasy"(MDMA) and related drugs. Canadian Medical Association Journal. CMAJ. 2001;2;165:917-28.

42. Campbell GA, Rosner MH. The agony of ecstasy: MDMA (3, 4-methylenedioxymethamphetamine) and the kidney. Clin J Am Soc Nephrol. 2008;3:1852-60. doi: 10.2215/ CJN.02080508.

43. De La Torre R, Farré M, Roset PN, Pizarro N, Abanades $S$, Segura M, et al. Human pharmacology of MDMA: pharmacokinetics, metabolism, and disposition. Ther Drug Monit. 2004;26:137-44.

44. Hall A, Henry J. Acute toxic effects of 'Ecstasy'(MDMA) and related compounds: overview of pathophysiology and clinical management. Br J Anaesth. 2006;96:678-85. doi: 10.1093/bja/ael078.

45. Bryden A, Rothwell P, O'Reilly P. Urinary retention with misuse of" ecstasy". Br Med J. 1995;310:504.

46. Kwon C, Zaritsky A, Dharnidharka VR. Transient proximal tubular renal injury following Ecstasy ingestion. Pediatr Nephrol. 2003;18:820-2. doi: 10.1007/s00467-003-1164-7.

47. Bora F, Yllmaz F, Bora T. Ecstasy (MDMA) and its effects on kidneys and their treatment: a review. Iran J Basic Med Sci. 2016;19:1151.

48. Song B-J, Moon K-H, V Upreti V, D Eddington N, J Lee I. Mechanisms of MDMA (ecstasy)-induced oxidative stress, mitochondrial dysfunction, and organ damage. Curr Pharm Biotechnol. 2010;11:434-43.

49. Ninković M, Selaković V, ĐUKIĆ M, Milosavljević P, Vasiljević I, Jovanović $M$, et al. Oxidative stress in rat kidneys due to 3, 4-methylenedioxymetamphetamine (ecstasy) toxicity. Nephrology (Carlton). 2008;13:33-7. doi: 10.1111/j.1440-1797.2007.00886.x.

Copyright (c) 2018 The Author(s); Published by Nickan Research Institute. This is an open-access article distributed under the terms of the Creative Commons Attribution License (http://creativecommons.org/licenses/by/4.0), which permits unrestricted use, distribution, and reproduction in any medium, provided the original work is properly cited. 